\title{
The Autonomy of Foreign Language Teachers and the Hidden Curriculum
}

\author{
Gao Ling-xin \\ Liren College, Yanshan University, Qinhuangdao, China
}

Email address:

gaolingxinglx@ysu.edu.cn

\section{To cite this article:}

Gao Ling-xin. The Autonomy of Foreign Language Teachers and the Hidden Curriculum. Science Journal of Education. Vol. 5, No. 4, 2017, pp. 153-157. doi: 10.11648/j.sjedu.20170504.15

Received: April 30, 2017; Accepted: May 13, 2017; Published: July 5, 2017

\begin{abstract}
There is the need to promote foreign language teachers' autonomy through the hidden curriculum in China. Hidden curriculum and teaching autonomy are two areas of educational research. From a unique perspective, this paper discusses the positive role that hidden curriculum plays for the promotion of teachers' autonomy. Through teachers' hidden curriculum, teachers' consciousness of innovation, emotion and reflection will do help for the promotion of the teaching effectiveness of foreign language teachers, and establishment of the good relationship between students and teachers, as well as the enhancement of teachers' self-development in their profession. Consequently, the goal of reforming the foreign language teaching will be achieved and teachers' autonomous teaching and students' autonomous learning will be realized. More than that, the strategic objectives of Chinese government educational reform to cultivate innovative talents will be integrated-during this process.
\end{abstract}

Keywords: Foreign Language Teachers, Hidden Curriculum, Teaching Autonomy

\section{Introduction}

The outline of the national medium and long term educational reform and development plan (2010-2020), which is referred to as the "Outline", clearly points out that "education should take the students as the main body and the teachers as the leading factor, and give full play to the initiative of the students... advocating heuristic, discovery, inquiry, discussion, participatory teaching, to help students gain the learning strategies.... Teachers should care for students... To affect the students with personality and charm of knowledge, make the healthy growth of the students and the guider." [1] "Outline" reflects the requirements for the three aspects of teachers, including the innovation of strategy, the devotion of emotion, and the consciousness of reflection, and these are the components of teachers' implicit class in the three aspects. Through the guidance and demonstration of teachers' implicit classroom, with diverse teaching strategies, can fully mobilize the enthusiasm of students, to stimulate students' learning motivation, through the whole process to maximize students' participation in learning. Another goal of the program is the realization of students' autonomous learning ability. Little (2004) believes that students' autonomous learning and teachers' autonomous teaching are interdependent, and teachers' teaching autonomy is the prerequisite for students to learn automatically [2]. Wang Shouren (2012) believes that teachers play a leading role in the settings of learning, content selection, teaching objectives, teaching design, teaching management and other aspects, to promote the teachers' ability of autonomous teaching as well as learning ability of students [3]. During teachers' self - teaching, the consciousness of innovation, emotion and reflection of teachers' hidden curriculum play an important role. By using the positive factors of hidden curriculum of teachers, teachers can consciously use the elements and change of curriculum, to construct reasonable curriculum consciously and purposefully, deepen the humanistic care for students, improve their professional development, optimize the allocation of resources in the autonomous teaching, promote the upgrading of teaching and to achieve the goal of cultivation of talents.

This paper explores the relationship between teachers' implicit classroom and teacher autonomy teaching, and helps to construct a new paradigm of foreign language teaching and research, which is embodied in the following aspects: First, the transition from the study of the language ontology 
and learners to study of teachers, reflects the rational regression of foreign language teaching in China. Secondly, the study on Teachers' hidden curriculum and study on teaching autonomy have become the focus of academic research, but study on the relationship between the two remained blank, so this paper is to explore a new perspective. In addition, independent teaching and recessive class teachers can achieve the goal of cultivating talent, which echoes the national aim in fostering innovative and speculative talents. In this paper, ways to improve teachers' teaching autonomy at three levels in the three dimensions of teachers' hidden curriculum will be discussed. Therefore, this paper, which studies the relationship between the two has a strong practical significance.

\section{Concept and Dimension}

According to quotation from "the International Encyclopedia of Education" by Fu Mingfu (2004), the Hidden Curriculum is defined as "various elements of informal learning of students, such as the relationship between teachers and students, grouping based on ability, classroom regulations and procedures, the metaphor of the textbook, students gender differences and curriculum reward mode" [4]. Although the hidden curriculum plays a subtle role in education for students, but for teachers, the hidden curriculum can be consciously used and transformed. Before the reasonable use of the hidden curriculum, the essential elements of the hidden curriculum should be classified. Fu Jianming (2000) divided the hidden curriculum into three layers respectively, such as the hidden curriculum in the textbooks, in addition to the teaching activities in the hidden curriculum and teaching activities in the environment of the hidden curriculum [5]; Jin Yule (1993) separate the unconsciously influence of the ideology and the process of curriculum implementation as one layer, another layer is the personality of teachers and teaching acts [6]; Ji Chengjun (2007) divided the Hidden Curriculum into two parts, one part is relevant to textbooks and the other part is related to the relationship between teachers and students [7]. The expression of these scholars affirmed the role of teachers as the hidden curriculum implementers. On the basis of the previous scholars' research, Shi Guangxiao (2011) [8] and Zeng Xiaoshan (2013) further refined the hidden curriculum on teachers' level and divided the different dimensions [9]. In this paper, based on the previous researches, the teacher curriculum ideology in carding, referring to Ceng Xiaoshan's classification on Teachers' recessive curriculum, this paper defines three dimensions of the hidden curriculum, which includes sense of responsibility, emotional awareness and sense of innovation, aiming at researching the relationship between teaching autonomy and hidden curriculum under the three dimensions.

The content of teachers' teaching autonomy is consistent with its dimensions. Chen Ying (2011) put forward seven levels of autonomy of English teachers, namely, teaching regulation, classroom activities, interest cultivation, emotional communication, student assessment, ability training, creative teaching [10]. Five kinds of teaching autonomy proposed by An Qi (2011) include teaching operation ability, teaching flexibility, professional development ability, the development of learner autonomy and insight, and effective teacher-student relationship [11]. Gao Jili, Li Xiuping (2010) defines six levels of autonomy, including the teaching plan, carrying out teaching activities and promoting learner autonomy ability, reflective ability, external relationship ability and self development capability [12]. Through the above analysis of the interpretation of scholars, teacher autonomy can be found on the existing theoretical level including level of classroom and level of teachers' psychological construction which has the intrinsic attribute characteristics. The level of classroom teaching effectiveness can be summarized as two aspects of the construction of teacher-student relationship and classroom effectiveness. While characteristics of teachers' level is mainly reflected in the ability of independent development level.

\section{Argument on the Relation}

\subsection{Enhance the Innovation Consciousness of Teachers}

The outline of the national medium - and long term educational reform and development plan (2010-2020) proposed the strategic theme of education in the next ten years. Huang Yuanshen (2010) believes that innovative English talents need to have strong analytical ability, speculative ability and independent thinking ability, ability to make full use of skilled English and knowledge in their field of innovation [13]. The cultivation of innovative talents requires teachers to have the innovative consciousness of teaching. Little's (2009) personal construction view holds the belief that "no matter how careful do the teachers follow the predetermined curriculum requirements, they are bound to pass their own unique views of the course [14]. When this kind of unique opinion is formed in the logical analytical reasoning, the comprehensive thinking, and the rational judgment, the innovation will be realized. The teaching autonomy under the consciousness of innovation is reflected in the unique understanding, creative development and reconstruction of individual teachers' teaching content and process. This sense of innovation to promote the teaching. Based on the teaching syllabus and teaching objects, teachers can make flexible selection and integration of teaching materials, adopt various teaching methods and promote innovation of students' thinking ability and learning ability, promote realization of the goals in foreign language teaching through the cultivation of innovative talents.

In the process of using teaching materials, teachers can use the hidden curriculum innovation consciousness, based on the characteristics of students, regional characteristics, teachers' knowledge structure and characteristics, teachers can take use of advanced audio and video, network and other media channels relevant to the content of the text, and further supplement, delete, innovate or refine the content; in addition, the design and organization of teaching can be enhanced through constant innovation, such innovative approach can be combined with the practical experience, the local characteristics of teachers, students and school, 
traditional and modern culture with specialized language of the times, through this way to expand and enrich the teaching content, to achieve the aim of efficient teaching. In view of the specific course design, it includes the teaching design of listening, speaking, reading, writing and translating, and also includes the design of a unit, a topic and a class. Dai Weidong (2008) believes that teachers' teaching methods should not be dogmatic, and a good teacher will be flexible to use all kinds of teaching methods to meet the needs of students [15]. According to the different aspects of listening, writing, translation, vocabulary learning, and different aspects to different cognitive styles, teachers can creatively adopt inspiring approach, integrated approach, audio lingual approach, deductive approach as teaching strategies. Besides, it should be combined with the topic and scenario, specific learning methods can be used for information channel, innovative role play and simulation interview and such kind of programs to promote the autonomy of students. Strengthen autonomous teaching through carrying out independent learning. Therefore, in the autonomous teaching, teachers also need to understand the role of innovation. According to Li Siqing (2011) Change the role of the traditional authority, to deal with different teaching links and different teaching content, in the teaching process to play a role in the auxiliary, supervision, guidance, questioning, or promotion [16]. For example, when teachers give instruction about difficult grammatical points, teachers should play the role of instructors to teach knowledge, when the content of the text is easy to understand, teachers can only appear as auxiliary cohesion in each link of teaching, and guide students as the main body to undertake content presentation and discussion. Under the guidance of innovation consciousness, teachers can expand the depth and breadth of teaching, at the same time, enhance students' interest in learning, enhance the learner's autonomous learning ability, and promote the realization of teaching objectives.

\subsection{Application of the Hidden Curriculum in the Classroom}

In the study of the relationship between Krashen's (1985) affective variables and second language acquisition, the affective variables of language learners include the following three parts: motivation, self-confidence and anxiety. The study found that individuals with high motivation, good selfconfidence and self-image, and low anxiety level tended to do better in the process of second language acquisition. Based on this theory, Krashen's (1985) affective filter is used to discuss the influence of affective factors on second language acquisition [17]. The study found that if second language learners do not have a positive emotional attitude, their language input is not only less, and even if they can understand the input information, this information will not reach up to the area of the brain which is responsible for language acquisition, learning effect is bad. An Qi (2011) pointed out that the harmonious relationship between teachers and students can help reduce the crisis and create an environment conducive to learning [18]. Therefore, it is necessary to attach importance to the role of teachers to improve their positive attitude. The relationship between the teacher and the students is reflected in Chen Jianlin's (2010) teaching ecology. He thinks that the foreign language teaching is a system, which is a natural and open ecological whole system, composed of the elements and the environment in a certain space [19]. In the whole ecosystem of foreign language teaching, the elements of classroom, teaching materials, students and teachers are integrated. Therefore, the emotional awareness of teachers in the classroom will play an important role in the relationship between teachers and students in teaching. A good relationship between teachers and students can promote the implementation of teaching autonomy, the tension between the teachers' teaching plan is difficult to implement smoothly. According to China's education reform document quoted by Wang Jing (2010), teachers and students in the classroom have a high degree of distance, the teacher is regarded as an authority, and the distance between teachers and students is large [20]. When teachers show respect and trust to the students, and encourage them, the distance between them is reduced. The students have been inspired, and will also to obtain a strong self-esteem. As An Qi (2011) said, strengthen the relationship between teachers and students the students' self-esteem will be enhanced. When the self-esteem of students enhanced, their learning initiative will be strengthened, and their independent learning ability will be improved accordingly [21]. Therefore, teachers should improve the emotional awareness in the classroom, increase students' self-confidence as language learners, and control their anxiety to improve the teaching effectiveness. According to a study of An Qi (2011), teaching ability of teachers, classroom control flexibility, respect for students, sense of humor, and knowledge are all the determining factors in harmonious relationship between teachers and students [22]. In addition, teachers by strengthening the body of their behavior, including pronunciation, intonation, facial expressions, movements, writing, body language and other aspects, can promote the expression of their emotions, and increase the way for students to exchange of information and emotional communication, enhance the teaching effectiveness. When teachers raise emotional awareness in the hidden curriculum, fully respect the subject status of both teachers and students through the implementation of the curriculum, and create the language learning scenes, comfort and stimulate the students' thinking and understanding based on their different emotional need. The emotion in the hidden curriculum will be able to completely penetrated, teacher will exchange ideas and share experiences with students in depth. Besides, through emotional interaction, students' interest and enthusiasm will be aroused, and the teacher-student relationship will be more harmonious.

\subsection{Strengthening the Reflective Consciousness of Foreign Language Teachers}

Wu Yian (2005) believes that the framework of professional qualities of foreign language teachers is 
composed of four dimensions: foreign language teaching ability, foreign language teachers' views about career and professional ethics, foreign language teaching views, as well as foreign language teachers' views in learning and development [23]. The development of foreign language teachers' view is the great impetus of its progress, this concept of development can be realized through the reflection of teaching in hidden curriculum, and sustained and in-depth reflection can promote teachers' teaching ability to improve teachers' self-development, implementation. The teacher's reflection on teaching is teachers' self-explanation, analysis, evaluation, reasoning and explanation, the purpose of which is to find the advantages and disadvantages of teaching, promote understanding and self-achievement, selfcorrection and provide guidance for the future teaching. In Zeng Xiaoshan's (2013) study, the teachers, who teach effectively, is through constant reflection and understanding of the basic problems of education and teaching, through such process, they formed a complete view on education, correct understanding, and they can grasp the laws in language learning and achieve their own professional growth [24]. This reflection is mainly reflected in three aspects:

(1) The teachers are active in supplement of knowledge once they are aware of their lack of knowledge. Feng Youlan (2004) believes that during the process of thinking or talking problems, such thinking and talking about themselves are ongoing absorb of knowledge, self-reflection is to "think" the thinking process. [25] Reflection in teaching practice is a reflection of the teaching activities, through the reflection on the class design, course development, and course implementation, teachers will make active construction of curriculum resources, modifying teaching goals, integrating internal and external resources, and improve the development of curriculum theory, promote curriculum practice, and further enhance the teaching ability.

(2) Research is another pillar of teacher reflection in hidden curriculum. Liu Runqing (2003) pointed out that the excellent teachers can combine teaching and scientific research; gain inspiration by teaching, by means of inspiration to do the project, through the project and experiments to verify the theory [26]. At the same time, scientific research can keep teachers' interest in teaching, ensure the teaching content and strategy remain scientific, forward-looking and following the requirements of the times. In teaching reflection, teachers who lack in view of the problems, can obtain professional knowledge in teaching and promote their own development. With the help of scientific research, in-depth analysis and practical research, they can draw the conclusion, overcome difficulties, enhance their knowledge level and teaching ability.

(3) The reflection in teaching practice is not only from self-correction, but also from the support of teamwork. On the issue of self-development, Richards and Farrel (2005) place particular emphasis on the teachers supporting group, peer counseling, group teaching approach [27]. Through the collaborative cooperation, resource sharing, teachers must reflect through mutual thinking and undergo systematic reflection, the reflection of the way includes writing reflective journals, listen to other teachers' classroom, video recording and analysis, and teaching action research. According to Yang Fuqing (2008), the cycling reflection process includes the steps of finding problems, analyzing causes, formulating solutions, and checking solutions [28], during this process, teachers will enhance the independent teaching ability and teaching efficiency, and the same time promote teachers' self-development.

\section{Conclusion}

Wen Qiufang (2008) believes that in the training of innovative talents in foreign languages, the teacher is the inspiration and the executor of innovative education [29]. Gong Yafu (2007) believes that teachers are important curriculum resources, as well as an important curriculum creator [30]. Therefore, foreign language teachers must make full use of the positive factors of the hidden curriculum, improve the teaching quality, realize the development of teaching autonomy, and promote the realization of the goals of foreign language teaching reform. In the promotion of the hidden curriculum innovation consciousness, teachers can consciously use the elements and the change of curriculum, teaching content and achieve the optimal allocation of resources, improve the students' enthusiasm for learning and promote teaching efficiency. And by strengthening teachers' implicit classroom emotional awareness, enhance the emotional interaction, increase deep experiences and promote cooperation between teachers and students to create a harmonious relationship between teachers and students selfteaching, promote the smooth implementation of the classroom learning and improve the learning effectiveness. In addition, by strengthening the reflective consciousness of teachers' hidden curriculum, teachers' self-development will be realized. Therefore, the implicit teaching in the classroom can significantly improve the students' language ability, emotional communication ability and autonomous learning ability. At the same time, through using the positive elements of the hidden curriculum, teachers will realize the improvement of teaching autonomy and the improvement of teaching efficiency.

\section{Foundation Item}

Project supported by Qinhuangdao science and technology program in 2016, item number: 201602A314, by GAO Lingxin (Liren College of Yanshan University).

\section{References}

[1] State Council. National medium and long term education reform and development program (2010-2020) [Z]. people's publishing house, 2010.

[2] Little, D. Learner autonomy, teacher and the Language Portolio. (for conference) [C]. 2004. 
[3] Wang Shouren. The Construction of Tteachers' Tteam in the Ceourse of Ceonstructing the College English Curriculum System [J]. Foreign Language Community, 2012 (4): 2-5.

[4] Fu Mingfu. "Hidden Curriculum of University and Talent Development" [M]. Guangzhou: Jinan University press, 2004.

[5] Fu Jianming. Hidden curriculum analysis [J]. Ceourse, Teaching Materials, Teaching Methods, 2000 (8): 55-59.

[6] Jin Yu Le. On the Potential Courses [J]. Ceurriculum Teaching Materials -, 1993 (6): 48-51.

[7] Ji Chengjun. University Course Introduction [M]. Shanghai Education Press, 2007.

[8] Shi Guangxiao. College English Curriculum Design and Rresearch from the Perspective of Hidden Curriculum [D]. Shanghai: Shanghai International Studies University, 2011.

[9] Zeng Xiaoshan. Sstudy of Teachers' Implicit Ceurriculum during the Implementation of College English Curriculum [D]. Shanghai: Shanghai International Studies University, 2013: 124a.

[10] Chen Ying. The Relationship between Teacher Autonomy and Learner Autonomy [J]. Foreign Language Teaching, 2011a (3): 55-60.

[11] An Qi. Teacher Autonomy in Foreign Language Teaching and Student Autonomy [M]. Beijing: Economic Science Press, 2011a.

[12] Gao Gili, Li Xiuping. Study on Teacher's Independent Research in the Field of Second Language Acquisition in Foreign Countries [J]. Journal of Primary and Secondary School English Teaching and Research, 2010 (6): 4-9.

[13] Huang Yuanshen. English Major Curriculum Must be Completely Reformed - "Talk about Absence of Thinking" [J]. Foreign language, 2010 (1): 11-16.

[14] Little, D. Autonomous learning methods and ways [M]. Translated by Qiu Yongzhong. Fuzhou: Fujian Education Press, 1991/2009.

[15] Dai Weidong. Foreign Language Education Development Report (1978-2008) [M]. Shanghai: Shanghai Foreign Language Education Press, 2008.

[16] Li Siqing. Research on Foreign language Teachers' Teaching Autonomy [D]. Shanghai: Shanghai Foreign Studies University, 2011.

[17] Krashen, S. D. The Input Hypothesis: Issues and Implications [M]. London: Longman, 1985.
[18] An Qi. Teacher Autonomy and Student Autonomy in Foreign Language Teaching [M]. Beijing: Economic Science Press, $2011 b$.

[19] Chen Jianlin. Integration of Computer Network and Foreign Language Curriculum - A Study Based on College English Teaching Reform $[\mathrm{M}]$. Shanghai Foreign Language Education Press, 2010.

[20] Wang Jing, Zhang Ailin. A Comparative Study on the Distance of Chinese and American College Students [J]. Journal of Chongqing University of Posts and Telecommunications, 2012 (9): 153-155.

[21] An Qi. Empirical Research on Foreign Language Teachers' Independent Teaching. Foreign language Teaching, 2011 (7): 51.

[22] An Qi. Teacher Autonomy and Student Autonomy in Foreign Language Teaching [M]. Beijing: Economic Science Press, 2011c.

[23] Wu Yian. Excellent Foreign Language Teachers Professional Quality [J]. Foreign Language Teaching and Research, 2005 (3): 199-204.

[24] Zeng Xiaoshan. Study of Teachers' Implicit Curriculum during the Implementation of College English curriculum [D]. Shanghai: Shanghai International Studies University, 2013b.

[25] Feng Youlan. Brief History of Chinese Philosophy [M]. Beijing: New World Press, 2004.

[26] Liu Runqing, Dai Manchun. Research on Current state and Development Strategy of Foreign Language Teaching Reform in Chinese Universities [M]. Beijing: foreign language teaching and research press, 2003.

[27] Richards, J \& Farrel, T. Professional Development for language Teachers Strategies for Teacher Learning [M]. London: Cambridge University Press, 2005: 202.

[28] Yang Fuqing. Self-Awareness, Self-Action, Self-Management [J]. Education Exploration, 2008: 98.

[29] Wen Qiufang. Research and Practice of Systematic Training of Innovative Talents among English Majors [A]. Beijing: Foreign Language Teaching and Research Press, 2008: 211.

[30] Gong Yafu. The Present Situation of Modern Foreign Language Teaching in China and Some Ideas on Foreign Language Teaching Evaluation [J]. Chinese English Course Network, 2007. 